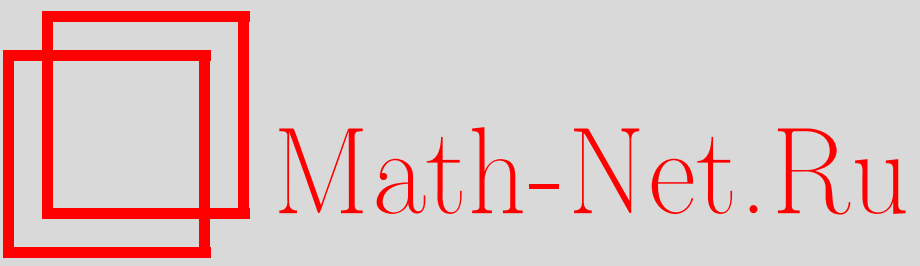

Н. В. Филимоненкова, Геометрия $m$-гессиановских уравнений, Итоги науки и техн. Сер. Соврем. мат. и ее прил. Темат. обз., 2019, том 169, 98-115

DOI: https://doi.org/10.36535/0233-6723-2019-169-98-115

Использование Общероссийского математического портала Math-Net.Ru подразумевает, что вы прочитали и согласны с пользовательским соглашением

http://www.mathnet.ru/rus/agreement

Параметры загрузки:

IP: 54.162 .27 .143

26 апреля 2023 г., 13:34:13 


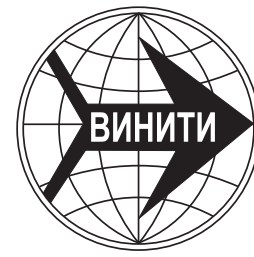

ИТОГИ НАУКИ И ТЕХНИКИ.

Современная математика и ее приложения.

Тематические обзоры.

Том 169 (2019). С. 98-115

DOI: $10.36535 / 0233-6723-2019-169-98-115$

УДК 514.763 .85

\title{
ГЕОМЕТРИЯ $m$-ГЕССИАНОВСКИХ УРАВНЕНИЙ
}

\author{
(c) 2019 г. $\quad$ Н. В. ФИЛИМОНЕНКОВА
}

\begin{abstract}
АннотАция. В процессе развития современной теории полностью нелинейных дифференциальных уравнений в частных производных второго порядка естественным образом появились новые геометрические характеристики поверхностей. Реализация этих характеристик на языке классической дифференциальной геометрии приводит к неоправданным техническим трудностям, близким к непреодолимым. Данная работа содержит обзор предпринятой методологической реформы и демонстрирует новый дифференциально-геометрический аппарат на примере построения приграничных барьеров для $m$-гессиановских уравнений.
\end{abstract}

Ключевъе слова: матрица кривизны, $p$-кривизна, $m$-выпуклая гиперповерхность, $m$-гессиановские уравнения, ядро приграничного барьера.

\section{GEOMETRY OF $m$-HESSIAN EQUATIONS}

\author{
(c) 2019 N. V. FILIMONENKOVA
}

\begin{abstract}
In the process of developing the modern theory of fully nonlinear, second-order partial differential equations, new geometric characteristics of surfaces naturally appeared. The implementation of these characteristics in terms of the classical differential geometry leads to significant technical difficulties. This paper provides a review of the necessary methodological reform and demonstrates a new differential geometric techniques by an example of constructing boundary barriers for $m$-Hessian equations.
\end{abstract}

Keywords and phrases: curvature matrix, $p$-curvature, $m$-convex hypersurface, $m$-Hessian equations, kernel of the boundary barrier.

AMS Subject Classification: 53A55, 35J60

1. Введение. Тема этой работы принадлежит области дифференциальной геометрии, но мотивирована теорией полностью нелинейных уравнений в частных производных второго порядка (FNPDE - fully nonlinear partial differential equations). Полностью нелинейными называют уравнения, нелинейные по вторым производным решения (в отличие от квазилинейных). Начало развитию современной теории FNPDE положили работы $[9,11,18]$, в которых построены локальные априорные оценки гельдеровских норм вторых производных решений. Эти результаты позволили использовать методы теории линейных эллиптических и параболических уравнений для доказательства разрешимости задачи Дирихле для уравнения Монжа-Ампера $\operatorname{det} u_{x x}=f$, родственных ему гессиановских уравнений и др. при условии $C^{2}$-априорной ограниченности решений.

До 1970 г. уравнение Монжа-Ампера было предметом исследования геометров (см. список источников в [10]); было показано, что задача Дирихле для него корректна в конусе выпуклых функций в выпуклых областях. В 1985 г. Каффарелли, Ниренберг и Спрук (см. [16]) предприняли попытку описать общий класс полностью нелинейных уравнений (включающий уравнение

Работа выполнена при поддержке Российского фонда фундаментальных исследований (проект № 18-01-00472). 
Монжа-Ампера), для которого задача Дирихле корректна в некотором конусе допустимых функций. С нашей точки зрения, принципиальные геометрические особенности новой теории содержит следующая теорема (см. [16, теорема 3, с. 264]):

Теорема 1. Задача Дирихле

$$
\begin{array}{ll}
\sigma_{m}\left(\lambda\left(u_{x x}\right)\right)=f>0 & \text { в } \bar{\Omega} \subset \mathbb{R}^{n}, m>1, \\
u=\varphi & \text { на } \partial \Omega
\end{array}
$$

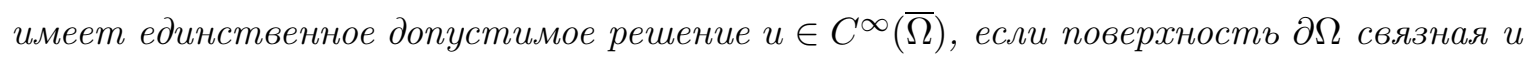

$$
\sigma_{m-1}\left(\kappa_{1}, \ldots, \kappa_{n-1}\right)>0 \quad \text { в каждой точке } x \in \partial \Omega .
$$

В случае $\varphi \equiv$ const условие (2) является такље необходимым для существования решения ${ }_{8} C^{2}(\bar{\Omega})$.

Здесь $\sigma_{m}$ - элементарная симметрическая функция порядка $m ; \lambda\left(u_{x x}\right)$ - собственные значения матрицы Гессе функции $u ;\left\{\kappa_{i}\right\}_{i=1}^{n-1}$ - главные кривизны гиперповерхности $\partial \Omega \subset \mathbb{R}^{n}$.

Уравнение (1) называется $m$-гессиановским. Оно полностью нелинейное при $m>1$. Семейство $m$-гессиановских уравнений, $m=2,3, \ldots, n$, содержит в качестве крайнего случая уравнение Монжа-Ампера $(m=n)$, и его можно дополнить линейным уравнением Пуассона $(m=1)$.

Условием (2) в теорию $m$-гессиановских уравнений была введена новая геометрическая характеристика гладких гиперповерхностей:

$$
\mathbf{k}_{p}=\sigma_{p}\left(\kappa_{1}, \kappa_{2}, \ldots, \kappa_{n-1}\right), \quad p=1,2, \ldots, n-1,
$$

известная как средняя кривизна при $p=1$ и гауссова кривизна при $p=n-1$. Характеристика $\mathbf{k}_{p}$ имеет решающее значение для построения локальных приграничных барьеров, однако в [16] это построение осуществляется неоправданно громоздкими средствами. Причина переусложненности заключается в том, что $m$-гессиановский оператор и величина $\mathbf{k}_{p}$ определяются в терминах собственных значений (как известно, главные кривизны - спектр оператора Вейнгартена). Такая форма записи эффектна, но не практична. Например, возникают не вызванные существом дела препятствия для дифференцирования уравнения $(1)$ : функция $\sigma_{m}\left(\lambda\left(u_{x x}\right)\right)$, будучи гладкой функцией точки $x$, оказывается выраженной через негладкие аргументы $\lambda\left(u_{x x}\right)$. С реализацией условия (2) на языке главных кривизн дело обстоит еще хуже: в классической дифференциальной геометрии отсутствует подходящий аппарат, и технические трудности становятся близки к непреодолимым. Данная методологическая проблема берет начало в [16] и до сих пор характерна для многих публикаций в этой области (например, [17, 28, 29]).

С 1980-х гг. исследованием $m$-гессиановских уравнений занимается Н. М. Ивочкина, причем уже в ранних работах $[2,3]$ развивает другой, более органичный подход к постановке и анализу задачи (1). Грубо говоря, элементарные симметрические функции от собственных значений матрицы (т.е. от корней характеристического многочлена) заменяются на суммы главных миноров этой матрицы (т.е. на коэффициенты многочлена). Для геометрического условия (2) это означает отказ от использования главных кривизн в качестве ведущих инвариантов поверхности и перенос внимания на новый инвариант - матрицу кривизны, которая является симметричным аналогом оператора (тензора) Вейнгартена. Эти простые и естественные идеи привели к пересмотру оснований дифференциальной геометрии и потому вызревали достаточно долго. Первые шаги были сделаны в [4], где впервые использована техника, позже названная инвариантным дифференцированием на поверхности, последние - в публикациях недавнего времени $[5,7,22,23]$ совместно с Н. В. Филимоненковой. В результате сложилась методология, в рамках которой характеристика $\mathbf{k}_{p}$, выраженная иным способом, называется $p$-кривизной и условие (2) наполняется следующим геометрическим смыслом: замкнутая поверхность $\partial \Omega$, удовлетворяющая неравенству $\mathbf{k}_{m-1}>0$, называется $(m-1)$-выпуклой.

В данной работе весь накопленный на этом пути дифференциально-геометрический аппарат синтезирован в одну систему согласованных инструментов исследования поверхностей и для примера коротко продемонстрировано его приложение к анализу задачи Дирихле для $m$-гессиановского уравнения (1). 
Статья носит обзорный характер. Утверждения, доказательства которых опубликованы ранее и не представляют интереса в развитии сюжетной линии, приводятся в виде формулировок со ссылкой на источники.

В статье используются следующие общепринятые обозначения:

$(x, y)$ - скалярное произведение векторов $x, y \in \mathbb{R}^{n} ; x^{2}=(x, x) ;|x|=\sqrt{(x, x)}$;

$C=\left(c_{j}^{i}\right), i=1,2, \ldots p, j=1,2, \ldots n$, - матрица размера $p \times n$ с элементами $c_{j}^{i}$;

$S=\left(s_{i j}\right)=\left(s_{i j}\right)_{i, j=1}^{n}$ - симметричная матрица размера $n \times n$ с элементами $s_{i j}$;

$O(n)$ - группа ортогональных $(n \times n)$-матриц;

$\theta_{\xi}=\left(\partial \theta^{i} / \partial \xi^{j}\right)$ - градиент вектор-функции $\theta(\xi) \in \mathbb{R}^{n}, \xi \in \mathbb{R}^{p}((n \times p)$-матрица Якоби $)$.

Символами $i, j, k, l, p, m, n, N$ по умолчанию обозначаются натуральные числа. Используется правило суммирования тензорного исчисления по повторяющимся верхним и нижним индексам.

2. Инвариантное дифференцирование. В этом разделе мы напоминаем понятие абсолютного геометрического инварианта поверхности и определяем инвариантное дифференцирование на поверхности.

Определение 1. Пусть $1 \leqslant n<N$. Связное множество $\Gamma^{n} \subset \mathbb{R}^{N}$ называется поверхностью коразмерности $N-n$, если существует такое число $r>0$, что для каждой точки $M_{0} \in \Gamma^{n}$ найдется область $\Theta \subset \mathbb{R}^{n}$ и гомеоморфизм $\Theta \rightarrow \Gamma^{n} \cap B_{r}\left(M_{0}\right)$, который будем называть локальной параметризацией поверхности (в окрестности данной точки $M_{0}$ ).

Свяжем с каждой точкой $M \in \Gamma^{n} \cap B_{r}\left(M_{0}\right)$ радиус-вектор $X(M) \in \mathbb{R}^{N}$ :

$$
X(\theta)=\left(x^{1}(\theta), x^{2}(\theta), \ldots, x^{N}(\theta)\right)^{T}, \quad \theta=\left(\theta^{1}, \theta^{2}, \ldots, \theta^{n}\right) \in \Theta \subset \mathbb{R}^{n} .
$$

Рассмотрим градиент $((N \times n)$-матрицу Якоби) этой вектор-функции:

$$
X_{\theta}=\left(\frac{\partial x^{i}}{\partial \theta^{j}}\right), \quad i=1,2, \ldots, N, \quad j=1,2, \ldots, n .
$$

Обозначим символами $X_{j}$ столбцы матрицы $X_{\theta}$ :

$$
X_{j}=\frac{\partial X}{\partial \theta^{j}}=\left(\frac{\partial x^{1}}{\partial \theta^{j}}, \frac{\partial x^{2}}{\partial \theta^{j}}, \ldots, \frac{\partial x^{N}}{\partial \theta^{j}}\right)^{T}, \quad j=1,2, \ldots, n .
$$

Как известно, векторы $X_{j}(M)$ являются касательными к поверхности $\Gamma^{n}$ в точке $M$. Метрическим тензором поверхности $\Gamma^{n}$ называется $(n \times n)$-матрица

$$
g(\theta)=X_{\theta}^{T} X_{\theta}, \quad g_{i j}=\left(X_{i}, X_{j}\right) .
$$

Взаимную однозначность соответствия между значениями параметра $\theta \in \Theta$ и точками $M \in$ $\Gamma^{n} \cap B_{r}\left(M_{0}\right)$ можно гарантировать условием

$$
\operatorname{det} g \neq 0 \text {. }
$$

Это условие, в свою очередь, равносильно тому, что векторы $\left\{X_{j}\right\}_{j=1}^{n}$ линейно независимы в каждой точке поверхности, а значит, образуют в касательной $n$-плоскости базис, вообще говоря, неортогональный.

Определение 2. Поверхность $\Gamma^{n} \subset \mathbb{R}^{N}$ называется $C^{k}$-гладкой, если в окрестности каждой точки она допускает $C^{k}$-гладкую локальную параметризацию (3), удовлетворяющую условию (6).

В статье по умолчанию рассматриваются $C^{k}$-гладкие поверхности, $k \geqslant 2$.

Если в окрестности данной точки $M_{0} \in \Gamma^{n}$ имеется хотя бы одна $C^{k}$-гладкая параметризация (3), удовлетворяющую условию (6), то их бесчисленное множество. Именно, любое $C^{k}$-гладкое отображение

$$
\xi=\left(\xi^{1}, \xi^{2}, \ldots, \xi^{n}\right) \in \Xi \subset \mathbb{R}^{n} \rightarrow \theta=\left(\theta^{1}, \theta^{2}, \ldots, \theta^{n}\right) \in \Theta \subset \mathbb{R}^{n}
$$

приводит к аналогичной параметризации, если $\operatorname{det} \theta_{\xi} \neq 0$. По знаку якобиана множество всех таких параметризаций разбивается на два класса эквивалентности: параметризации эквивалентны, 
если $\operatorname{det} \theta_{\xi}>0$. Локальные параметризации из одного, предпочтительного, класса эквивалентности назовем допустимыми.

Определение 3. Характеристика поверхности $\Gamma^{n}$ называется (абсолютным) геометрическим инвариантом, если она не зависит от выбора допустимой локальной параметризации.

Для поверхностей коразмерности 1 известно несколько абсолютных геометрических инвариантов: вектор нормали, нормальная кривизна поверхности в каком-либо направлении, главные кривизны и главные направления. Для поверхности произвольной коразмерности примером абсолютного геометрического инварианта является свойство линейной независимости касательных векторов $X_{j}$.

Дифференциальные характеристики (4), (5) геометрическими инвариантами не являются. При переходе к эквивалентной параметризации (7) они преобразуются по формулам

$$
X_{\xi}=X_{\theta} \theta_{\xi}, \quad g(\xi)=\theta_{\xi}^{T} g(\theta) \theta_{\xi} .
$$

Основные разделы данной работы посвящены описанию новых геометрических инвариантов, порожденных теорией FNPDE. В первую очередь это конструкция, которую мы называем «инвариантная производная».

Ключевую роль для этой конструкции играет $(n \times n)$-матрица $\tau$, определенная двумя следующими требованиями:

1) матрица $\tau$ должна удовлетворять разложению

$$
g^{-1}=\tau \tau^{T}
$$

2) должно выполняться естественное правило преобразования при замене (7) параметризации $X(\theta)$ на эквивалентную параметризацию $X(\xi)$ :

$$
\tau(\xi)=\theta_{\xi}^{-1} \tau(\theta)
$$

При $n>1$ требованиям (8), (9) удовлетворяет бесчисленное множество матриц $\tau$, и все они имеют вид

$$
\tau=\sqrt{g^{-1}} B, \quad B \in O(n) .
$$

Ортогональная матрица $B$ может быть выбрана произвольно, независимо от способа параметризации поверхности. Ее можно взять постоянной или переменной функцией точки на поверхности. Далее считаем, что выбор матрицы $B$ сделан и конкретная матрица $\tau$ вида (10) построена.

Отметим, что столбцы матрицы $\tau=\left(\tau_{j}^{i}\right)$, рассматриваемые как векторы $\tau_{j}=\left(\tau_{j}^{1}, \tau_{j}^{2}, \ldots \tau_{j}^{n}\right)^{T}$, $j=1,2, \ldots, n$, составляют ортонормированный базис на поверхности в смысле скалярного произведения $\left(g \tau_{i}, \tau_{j}\right)$.

Определение 4. Производные, вычисленные по правилу

$$
X_{(j)}=X_{k} \tau_{j}^{k}, \quad j=1,2, \ldots, n,
$$

назовем инвариантными. Векторы $X_{(j)}$ являются столбцами $(N \times n)$-матрицы $X_{(\theta)}=X_{\theta} \tau(\theta)$. Систему векторов $\left\{X_{(j)}\right\}_{j=1}^{n}$ назовем сопровождающим базисом поверхности $\Gamma^{n}$.

Векторы $X_{(j)}$ являются невырожденными линейными комбинациями касательных векторов $X_{j}$, поэтому они также принадлежат касательной $n$-плоскости поверхности $\Gamma^{n}$ в данной точке. Благодаря свойству (9) матрицы $\tau$ векторы $X_{(j)}$ не зависят от выбора допустимой локальной параметризации, для их вычисления подойдет любая:

$$
X_{(\xi)}=X_{\xi} \tau(\xi)=X_{\theta} \theta_{\xi} \theta_{\xi}^{-1} \tau(\theta)=X_{(\theta)} .
$$

Из свойства (8) матрицы $\tau$ вытекает, что $\left(X_{(i)}, X_{(j)}\right)=\delta_{i j}$. Таким образом, в отличие от базиса $\left\{X_{j}\right\}_{j=1}^{n}$, система $\left\{X_{(j)}\right\}_{j=1}^{n}$ является инвариантным ортонормированным базисом в касательных $n$-плоскостях. За счет выбора матрицы $\tau$ из семейства (10) в нашем распоряжении имеются ортогональные преобразования этого базиса. 
Введем обозначение для $(N \times n \times n)$-массива производных второго порядка вектор-функции $X(\theta)$ :

$$
X_{k l}=\frac{\partial^{2} X}{\partial \theta^{k} \partial \theta^{l}}, \quad X_{\theta \theta}=\left(X_{k l}\right)_{k, l=1}^{n} .
$$

Рассмотрим следующую коммутативную операцию:

$$
X_{(i j)}=X_{k l} \tau_{i}^{k} \tau_{j}^{l}, \quad X_{(\theta \theta)}=\left(X_{(i j)}\right)_{i, j=1}^{n}=\tau^{T} X_{\theta \theta} \tau .
$$

Вычислим инвариантные производные второго порядка:

$$
X_{(i)(j)}=\left(X_{(i)}\right)_{(j)}=\left(X_{k} \tau_{i}^{k}\right)_{l} \tau_{j}^{l}=X_{(i j)}+X_{k}\left(\tau_{i}^{k}\right)_{l} \tau_{j}^{l}, \quad X_{(\theta)(\theta)}=\left(X_{(i)(j)}\right)_{i, j=1}^{n} .
$$

Заметим, что производные (13) так же, как (11), инвариантны относительно выбора допустимой локальной параметризации, однако каждое из двух слагаемых выражения $X_{(i)(j)}$ по отдельности не инвариантно.

Инвариантное дифференцирование, как и классическое ковариантное, в общем случае не коммутативно, что приводит к естественным техническим трудностям. Исследование свойств коммутатора ждет внимания специалистов.

3. Матрица кривизны. В этом разделе описываем еще один новый геометрический инвариант, порожденный FNPDE, - матрицу кривизны для ориентированной гиперповерхности.

Начиная с этого раздела, полагаем $N=n+1$, т.е. рассматриваем гиперповерхности $\Gamma^{n} \subset$ $\mathbb{R}^{n+1}$, опуская иногда для краткости приставку «гипер». Пусть $M_{0} \in \Gamma^{n}$ и $X(\theta)$ - допустимая параметризация поверхности $\Gamma^{n} \cap B_{r}\left(M_{0}\right)$.

Как известно, единичный вектор нормали к поверхности можно вычислить (с точностью до направления) при помощи векторного произведения базисных векторов касательной $n$-плоскости:

$$
\mathbf{n}\left[\Gamma^{n}\right]=\frac{\left[X_{1}, X_{2}, \ldots, X_{n}\right]}{\left|\left[X_{1}, X_{2}, \ldots, X_{n}\right]\right|}=\left[X_{(1)}, X_{(2)}, \ldots, X_{(n)}\right] .
$$

Вектор нормали с выбранным направлением является абсолютным геометрическим инвариантом. Все характеристики гиперповерхности, которые вводим далее, осмысленны для двусторонних гиперповерхностей (в классическом значении этого термина, [1, гл. ХІІ]), ориентированных предпочтительным выбором направления $\mathbf{n}^{+}=\mathbf{n}^{+}\left[\Gamma^{n}\right]$. В случае замкнутой гиперповерхности мы отдаем предпочтение внутренней нормали, т.е. нормали, направленной в ограниченную область с краем $\Gamma^{n}$.

Допустим, что для поверхности $\Gamma^{n}$ зафиксирована матрица $\tau$ из семейства (10) и введено инвариантное дифференцирование (11). Заметим, что проекция вторых инвариантных производных (13) на инвариантный орт нормали выделяет в них коммутативные слагаемые (12). На основе этой идеи введем понятие матрицы кривизны.

Определение 5. Матрицей кривизны ориентированной гиперповерхности $\Gamma^{n}$ называем $(n \times$ $n)$-матрицу

$$
\begin{gathered}
\mathcal{K}\left[\Gamma^{n}\right]=\left(X_{(\theta)(\theta)}, \mathbf{n}^{+}\left[\Gamma^{n}\right]\right)=\tau^{T}(\theta)\left(X_{\theta \theta}, \mathbf{n}^{+}\left[\Gamma^{n}\right]\right) \tau(\theta), \\
\mathcal{K}_{i j}=\left(X_{(i)(j)}, \mathbf{n}^{+}\right)=\left(X_{k l}, \mathbf{n}^{+}\right) \tau_{i}^{k} \tau_{j}^{l}, \quad i, j=1,2, \ldots, n,
\end{gathered}
$$

где $\mathbf{n}^{+}$- орт внутренней нормали, если поверхность замкнутая.

Для незамкнутой поверхности есть две версии матрицы кривизны, соответствующие разному выбору $\mathbf{n}^{+}$.

Из формулы (14) ясно, что матрица кривизны является, во-первых, геометрически инвариантной и, во-вторых, симметричной.

Замечание 1. Если поверхность является $C^{k}$-гладкой и матрица $\tau$ выбрана $C^{k-2}$-гладкой функцией параметра $\theta$, то матрица (14) является $C^{k-2}$-гладкой функцией точки $M \in \Gamma^{n} \cap B_{r}\left(M_{0}\right)$. Напомним, что в качестве $\tau$ можно выбрать любую матрицу из семейства (10). Поэтому формула (14) на самом деле задает семейство матриц кривизны

$$
\mathcal{K}=B^{T} \mathcal{K}_{0} B, \quad B \in O(n),
$$


где матрица $\mathcal{K}_{0}$ соответствует выбору $\tau=\sqrt{g^{-1}}$. Поскольку $\sqrt{g^{-1}}$ несомненно является $C^{k-1}$ гладкой матрицей, то гладкость матрицы кривизны зависит от того, насколько гладкой выбрана ортогональная матрица $B=B(M)$.

Продемонстрируем частный случай определения 5. Рассмотрим замкнутую гиперповерхность $\Gamma^{1} \subset \mathbb{R}^{2}$, т.е. плоскую замкнутую кривую с какой-либо локальной параметризацией:

$$
X(\theta)=\left(x^{1}(\theta), x^{2}(\theta)\right)^{T}, \quad \theta \in \Theta \subset \mathbb{R}^{1} .
$$

Метрический тензор $g$ и матрица $\tau$ являются в данном случае числами, причем семейство $(10)$ состоит из одного элемента:

$$
X_{\theta}=\left(x_{\theta}^{1}, x_{\theta}^{2}\right)^{T} \Rightarrow g=X_{\theta}^{T} X_{\theta}=\left|X_{\theta}\right|^{2}=\left(x_{\theta}^{1}\right)^{2}+\left(x_{\theta}^{2}\right)^{2} \Rightarrow \tau=\sqrt{g^{-1}}=\frac{1}{\left|X_{\theta}\right|} .
$$

Очевидно, вектор

$$
\mathbf{n}^{+}=\frac{1}{\left|X_{\theta}\right|}\left(x_{\theta}^{1},-x_{\theta}^{2}\right)^{T}
$$

является единичной нормалью к кривой. Допустим, параметризация кривой такова, что данный вектор задает внутреннюю нормаль. Тогда матрица кривизны (14) вычисляется следующим образом:

$$
X_{\theta \theta}=\left(x_{\theta \theta}^{1}, x_{\theta \theta}^{2}\right)^{T} \Rightarrow \mathcal{K}\left[\Gamma^{1}\right]=\frac{x_{\theta \theta}^{1} x_{\theta}^{2}-x_{\theta \theta}^{2} x_{\theta}^{1}}{\left|X_{\theta}\right|^{3}} .
$$

Матрица кривизны плоской кривой есть число, которое мы называем кривизной этой кривой. В классической геометрии кривизной плоской кривой обычно называют модуль величины (15).

Для матрицы $\tau$ и для матрицы кривизны $\mathcal{K}$ существуют простые расчетные формулы в случае, когда поверхность $\Gamma^{n} \subset \mathbb{R}^{n+1}$ является графиком явно заданной функции:

$$
x^{n+1}=\omega(\tilde{x}), \quad \tilde{x}=\left(x^{1}, x^{2}, \ldots, x^{n}\right)^{T} .
$$

Введем обозначения:

$$
\begin{gathered}
\omega_{i}=\frac{\partial \omega}{\partial x^{i}}, \quad \omega_{\tilde{x}}=\left(\omega_{1}, \omega_{2}, \ldots, \omega_{n}\right), \quad \omega_{\tilde{x}} \times \omega_{\tilde{x}}=\left(\omega_{i} \omega_{j}\right)_{i, j=1}^{n} ; \\
\omega_{k l}=\frac{\partial^{2} \omega}{\partial x^{k} \partial x^{l}}, \quad \omega_{\tilde{x} \tilde{x}}=\left(\omega_{k l}\right)_{k, l=1}^{n} .
\end{gathered}
$$

Лемма 1. Предположим, что в окрестности некоторой точки гиперповерхность $\Gamma^{n}$ является графиком $C^{k}$-гладкой, $k \geqslant 2$, лвно заданной функиии (16). Тогда в качестве матрицы $\tau$ можно взять любую матричу вида

$$
\tau=\left(I-\frac{\omega_{\tilde{x}} \times \omega_{\tilde{x}}}{\sqrt{1+\omega_{\tilde{x}}^{2}}\left(1+\sqrt{1+\omega_{\tilde{x}}^{2}}\right)}\right) B, \quad B \in O(n) .
$$

Соответствующая матрица кривизны вычисляется по формуле

$$
\mathcal{K}= \pm \frac{\tau^{T} \omega_{\tilde{x} \tilde{x}} \tau}{\sqrt{1+\omega_{\tilde{x}}^{2}}}
$$

где знак «+» соответствуют выбору нормали $\mathbf{n}^{+}$, образующей острый угол с координатной осъю $x^{n+1}$.

Доказательство леммы 1 приведено в [8].

Приведем примеры вычисления матрицы кривизны по формулам (17), (18) для конкретных гиперповерхностей в пространстве $\mathbb{R}^{n+1}$. 
1. Для сферы (замкнутой поверхности) матрица кривизны однозначно определяется направлением внутренней нормали и является постоянной:

$$
x^{2}=R^{2} \quad \Rightarrow \quad x^{n+1}= \pm \sqrt{R^{2}-\tilde{x}^{2}} \quad \Rightarrow \quad \mathcal{K}=\frac{1}{R} I
$$

Заметим, что в этом идеальном случае матрица кривизны не зависит от выбора ортогональной матрицы $B$ в формуле (17).

2. Для гиперболоида (незамкнутой поверхности) матрица кривизны определена с точностью до знака, зависящего от ориентации, и с точностью до выбора ортогональной матрицы $B$ :

$$
x^{n+1}=\sqrt{\tilde{x}^{2}-R^{2}} \Rightarrow \mathcal{K}=\frac{ \pm 1}{\sqrt{2 \tilde{x}^{2}-R^{2}}}\left(I-\frac{2(B \tilde{x}) \times(B \tilde{x})}{2 \tilde{x}^{2}-R^{2}}\right) .
$$

Заметим, что формулы (17), (18) локально применимы к любой $C^{k}$-гладкой, $k \geqslant 2$, гиперповерхности $\Gamma^{n} \subset \mathbb{R}^{n+1}$ за счет выбора подходящей системы координат в евклидовом пространстве $\mathbb{R}^{n+1}$.

В завершение этого раздела опишем связь матрицы кривизны с классической дифференциальной геометрией, где в качестве старта для исследования кривизны ориентированных гиперповерхностей вводится геометрически инвариантное отношение второй и первой квадратичных форм.

Пусть $X(\theta)$ - допустимая локальная параметризация поверхности $\Gamma^{n}$. В какой-либо точке на поверхности рассмотрим дифференциал вектор-функции $X(\theta)$ :

$$
d X=X_{i} d \theta^{i} \in \mathbb{R}^{n+1}, \quad d \theta=\in \mathbb{R}^{n} .
$$

Вектор $d X$ принадлежит касательной $n$-плоскости и задает направление на поверхности. Вектор $d \theta$ является координатной записью этого направления в базисе $\left\{X_{i}\right\}_{i=1}^{n}$. Тогда

$$
(d X, d X)=(g d \theta, d \theta), \quad\left(d^{2} X, \mathbf{n}^{+}\right)=(b d \theta, d \theta)
$$

- соответственно первая и вторая квадратичные формы поверхности. Матрицей коэффициентов первой квадратичной формы является метрический тензор $g=X_{\theta}^{T} X_{\theta}$. Матрица второй квадратичной формы имеет вид $b=\left(X_{\theta \theta}, \mathbf{n}^{+}\right)$.

Нормальной кривизной гиперповерхности $\Gamma^{n}$ в направлении $d X$ в данной точке называется кривизна сечения $\Gamma^{n}$ двумерной плоскостью, натянутой на вектор нормали $\mathbf{n}^{+}$и касательный вектор $d X$. Формула

$$
\mathbf{k}(d \theta)=\frac{(b d \theta, d \theta)}{(g d \theta, d \theta)}, \quad d \theta \in \mathbb{R}^{n},
$$

выражает нормальную кривизну гиперповерхности $\Gamma^{n}$ в направлении $d X=X_{i} d \theta^{i}$.

Теперь рассмотрим в касательной $n$-плоскости вместо классического базиса $\left\{X_{i}\right\}_{i=1}^{n}$ ортонормированный сопровождающий базис $\left\{X_{(j)}\right\}_{j=1}^{n}$, состоящий из инвариантных производных векторфункции $X(\theta)$ (см. определение 4). После преобразования координат в формуле (21) получаем формулу

$$
\mathbf{k}(\delta \theta)=\frac{(\mathcal{K} \delta \theta, \delta \theta)}{|\delta \theta|^{2}}, \quad \delta \theta \in \mathbb{R}^{n},
$$

которая выражает нормальную кривизну гиперповерхности в направлении $d X=X_{(j)} \delta \theta^{j}$. Здесь использованы подстановка $d \theta=\tau \delta \theta$, свойство (8) матрицы $\tau$ и очевидная связь матрицы кривизны (14) с матрицей второй квадратичной формы: $\mathcal{K}=\tau^{T} b \tau$.

Итак, инвариантное дифференцирование позволило заменить представление (21) на эквивалентное (22).

Основными инвариантами классической геометрии поверхностей являются главные кривизны. Так называются стационарные значения нормальной кривизны (21) или, что то же самое, собственные значения несимметричной неинвариантной матрицы $g^{-1} b$ (тензор Вейнгартена). Из альтернативного представления (22) вытекает, что главные кривизны можно также вычислить как собственные значения матрицы $\mathcal{K}$, которая является инвариантным симметричным аналогом $g^{-1} b$. 
4. Конус Гординга. Дальнейший обзор новых геометрических инвариантов необходимо предварить небольшим алгебраическим отступлением.

В 1985 г. Кафферелли, Ниренберг и Спрук впервые привлекли к исследованию FNPDE алгебраическую теорию конусов Гординга (см. [16]). В [19] Гординг сформулировал понятие однородного $a$-гиперболического многочлена нескольких переменных как многочлена, имеющего только вещественные корни в направлении вектора $a$, и связал с такими многочленами специальные конусы (см. историко-математический обзор [14]). В [16] было обнаружено, что $a$-гиперболические многочлены порождают полностью нелинейные задачи, которые разрешимы в функциональных аналогах алгебраических конусов Гординга. Эталонным примером такой задачи является (1) в теореме 1: упомянутые допустимые функции и замкнутые гиперповерхности, удовлетворяющие условию (2), являются реализациями алгебраического конуса Гординга, который мы называем конусом $m$-положительных матриц.

Обозначим символом $\operatorname{Sym}(n)$ линейное пространство симметричных $(n \times n)$-матриц и символом $\lambda(S)=\left(\lambda_{1}, \lambda_{2}, \ldots, \lambda_{n}\right)$ набор собственных значений матрицы $S$.

Определение 6. Пусть $1 \leqslant p \leqslant n$. $p$-Следом матрицы $S \in \operatorname{Sym}(n)$ называется сумма всех главных миноров порядка $p$ матрицы $S$. Мы используем обозначение $T_{p}(S)$ (от англ. «trace») и полагаем $T_{0}(S) \equiv 1$. В частности, $T_{1}(S)=\operatorname{tr} S, T_{n}(S)=\operatorname{det} S$.

Рассмотрим характеристический многочлен матрицы $S$ в виде $\operatorname{det}(S+t I), t \in \mathbb{R}$. Из разложения

$$
\operatorname{det}(S+t I)=\sum_{p=0}^{n} T_{p}(S) \cdot t^{n-p}=\prod_{i=1}^{n}\left(t+\lambda_{i}\right), \quad \lambda_{i} \in \mathbb{R},
$$

и теоремы Виета следует, что p-след можно выразить как элементарную симметрическую функцию от собственных значений матрицы $S$ :

$$
T_{p}(S)=\sigma_{p}(\lambda(S))=\sum_{i_{1}<i_{2}<\ldots<i_{p}} \lambda_{i_{1}} \lambda_{i_{2}} \ldots \lambda_{i_{p}} .
$$

Представление (24) делает очевидной ортогональную инвариантность р-следов:

$$
T_{p}\left(B^{T} S B\right)=T_{p}(S), \quad B \in O(n) .
$$

Функция $T_{p}(S)$ порождает обобщение для понятия положительно определенной матрицы.

Определение 7. Пусть $1 \leqslant m \leqslant n$. Симметричную $(n \times n)$-матрицу $S$ называем $m$-положительной, если все ее $p$-следы положительны при $p=1,2, \ldots, m$.

Так как $T_{0}(S) \equiv 1$, то любая симметричная матрица по умолчанию является 0-положительной. При $m>1$ множество всех $m$-положительных матриц образует односторонний конус в пространстве $\operatorname{Sym}(n)$ :

$$
K_{m}=K_{m}(n)=\left\{S \in \operatorname{Sym}(n): T_{p}(S)>0, p=1,2, \ldots, m\right\} .
$$

Из разложения $(23)$ вытекает, что $K_{n}$ - это в точности конус положительно определенных матриц в пространстве $\operatorname{Sym}(n)$.

Итак, получаем следующую иерархию конусов (26):

$$
\operatorname{Sym}(n)=K_{0} \supset K_{1} \supset K_{2} \supset \cdots \supset K_{n} .
$$

Конусы (26) впервые определила и исследовала Н. М. Ивочкина в [3], еще до знакомства с теорией Гординга. Широкую известность среди специалистов эти конусы приобрели после публикации работы [16], в которой Кафферелли, Ниренберг и Спрук представили (26) как частный случай конусов Гординга. Сам термин «m-положительная матрица» для наименования элементов из $K_{m}$ введен недавно в $[5,26]$.

В любом конусе Гординга (см. [14]) справедливо свойство монотонности, которое для $K_{m}$ выглядит следующим образом:

$$
T_{m}(S+\tilde{S})>T_{m}(S), \quad S \in K_{m}, \quad \tilde{S} \in \bar{K}_{m} .
$$


Отсюда следует, что если $S \in K_{m}, \tilde{S} \in \bar{K}_{m}$, то $(S+\tilde{S}) \in K_{m}$. Из кососимметричности миноров вытекает равенство

$$
T_{m}(S+\xi \times \xi)=T_{m}(S)+T_{m}^{i j}(S) \xi_{i} \xi_{j}, \quad T_{m}^{i j}(S)=\frac{\partial T_{m}(S)}{\partial s_{i j}}, \quad \xi \in \mathbb{R}^{n} .
$$

Поскольку $\xi \times \xi \in \bar{K}_{n} \subset \bar{K}_{m}$, то (28) и (29) означают, что $T_{m}^{i j}(S) \xi_{i} \xi_{j}>0$ в $K_{m}$.

Согласно общей теории Гординга конус $K_{m}$ выпуклый и функция $F_{m}=T_{m}^{1 / m}$ вогнута в $K_{m}$. Вместе с 1-однородностью функции $F_{m}$ это приводит к соотношению

$$
F_{m}(S) \leqslant F_{m}^{i j}(\tilde{S}) s_{i j}, \quad S, \tilde{S} \in K_{m}, \quad F_{m}^{i j}(\tilde{S})=\frac{\partial F_{m}(\tilde{S})}{\partial \tilde{s}_{i j}}
$$

В дальнейшем нам понадобится следующее альтернативное описание конуса (26).

Лемма 2. Пусть матрица $S_{0} \in \operatorname{Sym}(n)$ является $m$-положительной. Конус $K_{m}$ совпадает c той компонентой связности множества $\left\{S \in \operatorname{Sym}(n): T_{m}(S)>0\right\}$, которая содержит матрииу $S_{0}$.

Простое доказательство этой леммы можно найти в [7, лемма 2.2].

Для положительно определенных, или $n$-положительных, матриц широко известен критерий Сильвестра. В $[7,13]$ доказан аналог критерия Сильвестра для $m$-положительных матриц.

Лемма 3 (критерий Сильвестра). Пусть $S \in \operatorname{Sym}(n), 1 \leqslant m \leqslant n$.

(i) Выберем некоторый номер $i, 1 \leqslant i \leqslant n$, и обозначим символом $S^{\langle i\rangle} \in \operatorname{Sym}(n-1)$ матричу, полученную из $S$ вычеркиванием строки и столбца с номером $i$. Тогда

$$
S \in K_{m}(n) \quad \Longleftrightarrow \quad T_{m}(S)>0, S^{\langle i\rangle} \in K_{m-1}(n-1) .
$$

(ii) Выберем некоторый набор попарно различных номеров $1 \leqslant i_{1}, i_{2}, \ldots, i_{m-1} \leqslant n$. Обозначим символом $S^{\left\langle i_{1}, i_{2}, \ldots, i_{k}\right\rangle} \in \operatorname{Sym}(n-k)$ матрииу, полученную из матрицы $S$ вычеркиванием $k$ строк и $k$ столбцов с указанными номерами. Чтобы матрица $S$ была $m$-положительной, необходимо и достаточно выполнения следующих условий:

$$
T_{m}(S)>0, \quad T_{m-1}\left(S^{\left\langle i_{1}\right\rangle}\right)>0, \quad T_{m-2}\left(S^{\left\langle i_{1}, i_{2}\right\rangle}\right)>0, \quad \ldots, \quad T_{1}\left(S^{\left\langle i_{1}, i_{2}, \ldots, i_{m-1}\right\rangle}\right)>0 .
$$

Замечание 2. Из произвольности выбора номеров $i_{1}, i_{2}, \ldots, i_{m-1}$ следует, что $m$-положительная матрица имеет по крайней мере $m$ положительных собственных значений (обратное верно только при $m=n)$.

5. Понятие $p$-кривизны и $m$-выпуклой гиперповерхности. Возвращаясь к обзору новых геометрических инвариантов, введем квалифицированное определение $p$-кривизны гиперповерхности. Допустим, для ориентированной гиперповерхности $\Gamma^{n} \subset \mathbb{R}^{n+1}$ выбрана матрица $\tau$ из семейства (10) и построена матрица кривизны $\mathcal{K}$ по формуле $(14)$.

Определение 8. Пусть $M \in \Gamma^{n}$. $p$-Кривизнами гиперповерхности $\Gamma^{n}$ в точке $M$ называем числа

$$
\mathbf{k}_{p}(M)=T_{p}\left(\mathcal{K}\left[\Gamma^{n}\right]\right)(M), \quad p=1,2, \ldots, n .
$$

По определению положим $\mathbf{k}_{0} \equiv 1$.

Свойство (25) p-следов делает $p$-кривизны не зависящими от того, какой выбрана матрица $\tau$ из семейства (10). С учетом замечания 1 это значит, что $p$-кривизны являются $C^{k-2}$-гладкими функциями точки на $C^{k}$-гладкой поверхности, $k \geqslant 2$.

Выражение $p$-кривизны через набор $\left\{\kappa_{i}\right\}_{i=1}^{n}$ главных кривизн гиперповерхности обусловлено равенством (24) и тем, что главные кривизны составляют спектр матрицы кривизны:

$$
\mathbf{k}_{p}=\sigma_{p}\left(\kappa_{1}, \kappa_{2}, \ldots, \kappa_{n}\right)=\sum_{i_{1}<i_{2}<\ldots<i_{p}} \kappa_{i_{1}} \kappa_{i_{2}} \ldots \kappa_{i_{p}}, \quad p=1,2, \ldots, n .
$$


Следовательно, средняя кривизна и гауссова кривизна включены в определение 8 как 1-кривизна и $n$-кривизна. Геометрический инвариант (2), который встречается в теореме 1 , в наших терминах называется $(m-1)$-кривизной гиперповерхности $\partial \Omega$.

Равенство (32) показывает, что наборы $\left\{\kappa_{i}\right\}_{i=1}^{n}$ и $\left\{\mathbf{k}_{p}\right\}_{p=1}^{n}$ полностью определяют друг друга, как корни и коэффициенты многочлена. Однако главные кривизны, в отличие от $p$-кривизн, не обладают глобальной нумерацией, являются, вообще говоря, трудно вычислимыми инвариантами, и какой бы гладкой ни была поверхность, можно гарантировать не более чем их липшицеву гладкость как функций точки на поверхности.

Замечание 3. С появлением понятия $p$-кривизн возникли новые задачи на стыке FNPDE и дифференциальной геометрии. Появились аналоги задач о построении поверхностей по заданной средней или гауссовой кривизне. Последняя, как известно, называется классической проблемой Минковского (см. [10]). Задача о построении замкнутой гиперповерхности по заданной $p$-кривизне, $1 \leqslant p<n-1$, сформулирована в монографии [10] и названа обобщенной проблемой Минковского; она не имеет удовлетворительного решения до сих пор. Построение поверхности по заданной $p$-кривизне и краю исследовалось в $[4,17,27]$, но также не доведено до конца. В $[6,20,21,25,30])$ получены результаты об эволюции замкнутых выпуклых гиперповерхностей с предписанным законом изменения $p$-кривизны, но остаются открытыми задачи о сжимающей эволюции $m$-выпуклых гиперповерхностей.

Связующим звеном между алгебраическими конусами (26) и дифференциальной геометрией является следующая классификация гладких гиперповерхностей.

Определение 9. Пусть $0 \leqslant m \leqslant n$ и $\Gamma^{n}-C^{k}$-гладкая ориентированная гиперповерхность, $k \geqslant 2$. Гиперповерхность $\Gamma^{n}$ называем $m$-выпуклой в точке $M \in \Gamma^{n}$, если матрица $\mathcal{K}[\Gamma](M)$ является $m$-положительной.

Гиперповерхность $\Gamma^{n}$ называется $m$-выпуклой, если она $m$-выпукла в каждой точке $M \in \Gamma^{n}$.

В соответствии с определением $7 m$-положительных матриц и определением $8 p$-кривизн множество всех $m$-выпуклых гиперповерхностей можно описать следующим образом:

$$
\mathbf{K}_{m}=\left\{\Gamma^{n} \subset \mathbb{R}^{n+1}: \mathbf{k}_{p}(M)>0, p=1,2, \ldots, m, M \in \Gamma^{n}\right\} .
$$

Это множество является конусом относительно умножения радиус-вектора поверхности на положительный коэффициент. Для этих конусов справедлива цепочка включений, аналогичная (27).

Все гладкие поверхности считаются 0-выпуклыми. Из замечания 2 следует, что $m$-выпуклая поверхность имеет по крайней мере $m$ положительных главных кривизн. Значит, $n$-выпуклая поверхность является строго выпуклой в классическом смысле. Однако понятие $n$-выпуклой поверхности исключает из рассмотрения границы строго выпуклых областей, которые вообще не являются гладкими или имеют изолированные точки нулевой гауссовой кривизны. В определении 9 -выпуклым поверхностям предписана положительная гауссова кривизна $\mathbf{k}_{n}$.

Определения 8, 9 являются однозначными только для замкнутых гиперповерхностей $\Gamma^{n}$, поскольку в этом случае при построении матрицы кривизны делается однозначный выбор в пользу внутренней нормали (см. раздел 3). Если поверхность $\Gamma^{n}$ незамкнутая, то как знаки главных кривизн, так и знак $p$-кривизны при нечетном значении $p$, зависят от выбора направления нормали. Поэтому и называть такую поверхность $m$-выпуклой можно с точностью до ориентации. Приведем примеры.

1. Сфера $x^{2}=R^{2}$ является $n$-выпуклой поверхностью, что очевидно по ее матрице кривизны (19). 2. Гиперболоид $x^{n+1}=\sqrt{\tilde{x}^{2}-R^{2}}, \tilde{x}=\left(x^{1}, x^{2}, \ldots, x^{n}\right)$, ориентированный нормалью, которая образует острый угол с координатной осью осью $x^{n+1}$, имеет матрицу кривизны (20) со знаком «+». Такой гиперболоид является всюду $m$-выпуклой поверхностью, если $m<n / 2$. Если $n / 2 \leqslant m<n$, то гиперболоид является $m$-выпуклым в точках, расположенных выше $n$-плоскости $x^{n+1}=\sqrt{(2 m-n) /(2 n-2 m)} R$.

Подробный анализ $m$-выпуклости многомерных квадрик (параболоидов и гиперболоидов) содержится в [15].

Простой критерий $m$-выпуклости вытекает из леммы 2. 
Лемма 4. Для того чтобы гиперповерхность $\Gamma^{n}$ была $m$-выпуклой, необходимо и достаточно существования хотя бы одной точки $M_{0} \in \Gamma^{n}$, в которой поверхность $\Gamma^{n} m$-выпукла, $u$ выполнения условия $\mathbf{k}_{m}(M)>0$ во всех точках $M \in \Gamma^{n}$.

Для того чтобы замкнутая гиперповерхность $\Gamma^{n}$ была $m$-выпуклой, необходимо и достаточно выполнения условия $\mathbf{k}_{m}(M)>0$ во всех точках $M \in \Gamma^{n}$.

Необходимость очевидна из определения 9, по существу важна достаточность. Первое утверждение леммы 4 есть прямой аналог леммы 2. Второе утверждение леммы 4 следует из первого, так как на замкнутой поверхности обязательно есть точка $n$-выпуклости.

Таким образом, для замкнутой поверхности $m$-выпуклость равносильна положительности ее $m$-кривизны. Следовательно, в теореме 1 утверждается разрешимость задачи Дирихле (1) в области с $(m-1)$-выпуклой границей.

6. Приложение новых геометрических инвариантов к FNPDE. Рассмотрим функцию $u \in C^{2}(\bar{\Omega})$, где $\Omega \subset \mathbb{R}^{n}$ - ограниченная область. Символом $u_{x}$ обозначен градиент функции $u$, символом $u_{x x}$ - матрица ее частных производных второго порядка (матрица Гессе). Пусть $1 \leqslant$ $m \leqslant n$. Рассмотрим задачу Дирихле для стационарного $m$-гессиановского уравнения:

$$
\begin{aligned}
& T_{m}[u]=T_{m}\left(u_{x x}\right)=f>0 \quad \text { в } \Omega, \\
& \left.u\right|_{\partial \Omega}=\varphi .
\end{aligned}
$$

Как уже было замечено во введении, в большинстве публикаций для определения $m$-гессиановского оператора вместо функции $T_{m}(S)$ используется $\sigma_{m}(\lambda(S))$ (теорема 1 ).

Очевидно, что решение задачи (33) для 1-гессиановского уравнения (уравнения Пуассона) надо искать среди функций $u$, у которых матрица Гессе обладает в области $\Omega$ положительным 1 следом. Как известно, задачу (33) для $n$-гессиановского уравнения (уравнения Монжа-Ампера) целесообразно рассматривать на множестве строго выпуклых функций. В поисках естественного множества разрешимости для задачи (33) при $1<m<n$ Н. М. Ивочкина в [3] построила матричный конус $K_{m}$ (см. определение 7 , формулу $(26)$ ) и его функциональный аналог:

$$
\mathbb{K}_{m}(\Omega)=\left\{u \in C^{2}(\Omega): T_{p}[u](x)>0, p=1,2, \ldots, m, x \in \Omega\right\} .
$$

После публикации [16] 1985 г., в которой Кафферелли, Ниренберг, Спрук ввели термин «допустимая функция» для широкого класса FNPDE, элементы функционального конуса $\mathbb{K}_{m}(\Omega)$ мы называем $m$-допустимыми функциями.

Определение 10. Функция $u \in C^{2}(\Omega)$ называется $m$-допустимой в точке $x \in \Omega$, если матрица $u_{x x}$ является $m$-положительной в этой точке. Функция $u$ называется $m$-допустимой в области $\Omega$, если она $m$-допустима в каждой точке $x \in \Omega$.

Все $C^{2}$-гладкие функции считаются 0-допустимыми и образуют конус $\mathbb{K}_{0}(\Omega)$. Любая $n$-допустимая функция является строго выпуклой в классическом смысле, поскольку ее матрица Гессе положительно определена. Обратное может быть неверно, поскольку классическое определение строгой выпуклости распространяется на негладкие функции и на функции, имеющие точки вырождения матрицы Гессе. Для конусов $\mathbb{K}_{m}(\Omega)$ справедлива цепочка включений, аналогичная $(27)$.

Заметим, что согласно определению 10 функция $u$ не может иметь точек максимума в области m-допустимости.

Сформулируем простое следствие леммы 2.

Лемма 5. Пусть $u \in C^{2}(\Omega), 1 \leqslant m \leqslant n$. Для того чтобы функиия и была $m$-допустимой в области $\Omega$, необходимо и достаточно существования хотя бы одной точки $x_{0} \in \Omega$, в которой $u_{x x}\left(x_{0}\right) \in K_{m}$, и выполнения условия $T_{m}[u]>0$ во всех точках $x \in \Omega$.

В частности, условие $T_{m}[u]>0$ гарантирует $m$-допустимость функции $u$, достигающей минимума в $\Omega$.

Определение 10 и лемма 5 , сформулированные для открытой области $\Omega$, дословно переносятся на замкнутую область $\bar{\Omega}$. 
Нельзя не заметить аналогию между конусом $m$-допустимых функций $\mathbb{K}_{m}(\Omega)$ (определение 10 ) и конусом $m$-выпуклых гиперповерхностей $\mathbf{K}_{m}$ (определение 9 ). И тот, и другой определяются на основе конуса $m$-положительных матриц $K_{m}$ (определение 7 ) и при $m=n$ приводят к строго выпуклым функциям и поверхностям соответственно.

Поясним, почему функции $u \in \mathbb{K}_{m}(\Omega)$ называются $m$-допустимыми, а не, скажем, $m$-выпуклыми. Дело в том, что $m$-выпуклыми целесообразно было бы называть функции, график которых является $m$-выпуклой гиперповерхностью в смысле определения 9 . Для функций из конуса $\mathbb{K}_{m}(\Omega)$ при $m<n$ это не так. Однако верно следующее утверждение.

Лемма 6. Пусть $u \in C^{2}(\bar{\Omega}), 1 \leqslant m \leqslant n$. Предположим, что

$$
T_{m}[u]>0, \quad x \in \bar{\Omega},\left.\quad u\right|_{\partial \Omega}=\text { const } .
$$

Тогда

$$
\left.u_{x}\right|_{\partial \Omega} \neq \mathbf{0}, \quad \partial \Omega \in \mathbf{K}_{m-1} .
$$

В частности, невырожденная поверхность уровня $m$-допустимой функиии является $(m-1)$ выпуклой гиперповерхностъю.

Доказательство этой леммы приведено в [7, теорема 4.1]. Другие свойства $m$-допустимых функций разбираются в [22]. Понятие $m$-допустимой функции для эволюционного $m$-гессиановского оператора введено в $[7,24]$.

В [12] проведено полное исследование классической и слабой (аппроксимативной) разрешимости задачи (33) в конусе $m$-допустимых функций, построена шкала зависимости гладкости решения от гладкости данных задачи. В частности, получена следующая уточненная версия теоремы 1.

Теорема 2. Пусть $\varphi \in C^{l+\alpha}(\partial \Omega), f \in C^{l-2+\alpha}(\bar{\Omega}), \partial \Omega \in C^{l+\alpha}, f>0$ в $\bar{\Omega}, l \geqslant 4,0<\alpha<1$. Пусть, кроме того,

$$
\mathbf{k}_{m-1}[\partial \Omega]>0
$$

Тогда существует единственное т-допустимое решение $u \in C^{l+\alpha}(\bar{\Omega})$ задачи (33).

Напомним, что согласно лемме 4 условие (36) равносильно тому, что замкнутая гиперповерхность $\partial \Omega$ является $(m-1)$-выпуклой.

Рассмотрим теорему 2 для $\varphi \equiv c, c \in \mathbb{R}$. Из лемм 5 и 6 следует, что в этом случае требование (36) необходимо для существования не только $m$-допустимого решения, но и любого $C^{2}$ гладкого решения задачи Дирихле (33). При этом, если $c \neq 0$ или число $m$ нечетное, существует только одно $C^{2}$-гладкое решение задачи (33) и оно $m$-допустимо. Если же $c=0$ и $m$ четно, то задача (33) имеет два $C^{2}$-гладких решения: $u$ и $-u$, и одно из них $m$-допустимо.

Поясним идею доказательства теоремы 2 (подробнее см. [12]). Соотношения (28), (29) гарантируют эллиптичность оператора $T_{m}[u]$ на каждой функции $u \in \mathbb{K}_{m}(\Omega)$ и равномерную эллиптичность на решении (33) при условии $f>0$ в $\bar{\Omega}$ и при наличии априорной $C^{2}$-оценки решения в области $\bar{\Omega}$. Принцип максимума Александрова «вытесняет» $C^{2}$-оценки решения на границу области $\Omega$.

Ключевой и наиболее трудоемкой частью доказательства теоремы 2 является получение априорных $C^{2}$-оценок решения вблизи $\partial \Omega$. Для этого используется особый способ построения $m$-гессиановских приграничных нижних барьеров, отличный от техники, которую использовали Кафферелли, Ниренберг, Спрук для задачи (1) в [16]. Предпосылки данных барьеров можно найти в [4]; в развитом виде они используются в работах последних лет Н. М. Ивочкиной и Н. В. Филимоненковой.

В завершение данной работы опишем наше построение барьеров с наиболее общей точки зрения, чтобы продемонстрировать использование новых дифференциально-геометрических инструментов.

Докажем сначала принцип сравнения для 1-однородной модификации $m$-гессиановского оператора:

$$
F_{m}[u]=\left(T_{m}[u]\right)^{1 / m}
$$


Точнее, будем рассматривать оператор $F_{m}$ в рамках более широкого класса псевдолинейных эллиптических операторов:

$$
F_{m}^{i j}[u] v_{i j}, \quad F_{m}^{i j}[u]=\frac{\partial F_{m}\left(u_{x x}\right)}{\partial u_{i j}},
$$

где $u-m$-допустимая функция, $v$-произвольная функция (в том числе может быть $v=u$, и тогда $\left.F_{m}^{i j}[u] u_{i j}=F_{m}[u]\right)$.

Лемма 7. Пусть $u \in \mathbb{K}_{m}(\Omega), v \in C^{2}(\Omega), \mu>0$ и в каждой точке $x \in \Omega$

$$
F_{m}^{i j}[u] v_{i j} \leqslant \mu .
$$

Пусть имеется такая функиия $w \in \mathbb{K}_{m}(\Omega)$, что в каждой точке $x \in \Omega$

$$
F_{m}[w] \geqslant \mu .
$$

Тогда

$$
v(x)-w(x) \geqslant \min _{\partial \Omega}(v-w), \quad x \in \Omega .
$$

Доказательство. Допустим, что функция $(v-w)$ достигает минимума в точке $x_{0} \in \Omega$. Тогда в точке $x_{0}$ матрица $\left(v_{x x}-w_{x x}\right)$ неотрицательно определена, т.е. $\left(v_{x x}-w_{x x}\right)\left(x_{0}\right) \in \bar{K}_{n}$. По условию имеем $w_{x x} \in K_{m}$. Из монотонности $(28)$ вытекает, что $v_{x x}\left(x_{0}\right) \in K_{m}$. Пользуясь вогнутостью функции $F_{m}$ в конусе $K_{m}$ (неравенство (30)) и еще раз монотонностью $(28)$, получаем в точке $x_{0}$

$$
F_{m}^{i j}[u] v_{i j} \geqslant F_{m}[v]=F_{m}[w+v-w]>F_{m}[w] .
$$

Это противоречит условиям (37), (38).

Поскольку в конусе $\mathbb{K}_{m}(\Omega)$ справедливо неравенство вогнутости $(30)$, то $F_{m}^{i j}[u] w_{i j} \geqslant F_{m}[w]$, и лемма 7 является $m$-гессиановской версией классического принципа максимума для линейных эллиптических уравнений в частных производных второго порядка.

Функцию $w$ принято называть нижним баръером для функции $v$.

При $v=u$ условия (37), (38) можно записать проще:

$$
T_{m}[u] \leqslant \mu \leqslant T_{m}[w] .
$$

В этом случае функция $w$ служит нижним барьером для функции $u$, которая может быть, в частности, решением уравнения (33) с ограниченной правой частью. В более общем неравенстве (37) на роль $v$ подходит не только решение задачи (33), но и, скажем, его производные.

Нижний барьер $w$, специальным образом локализованный в окрестности точки $M_{0} \in \partial \Omega$, дает оценку снизу для производной по нормали функции $v$.

Свяжем с точкой $M_{0} \in \partial \Omega$ приграничную область:

$$
\Omega_{r} \subset \Omega \cap B_{r}\left(M_{0}\right), \quad \partial \Omega_{r} \cap \partial \Omega=B_{r}\left(M_{0}\right) \cap \partial \Omega, \quad 0<r \ll 1 .
$$

Следствие 1. Предположим, что найдется область $\Omega_{r}$ вида (39), в которой выполнены все условия леммы 7 и, кроме того,

$$
v\left(M_{0}\right)=w\left(M_{0}\right),\left.\quad(v-w)\right|_{\partial \Omega_{r}} \geqslant 0 .
$$

Тогда

$$
\frac{\partial v}{\partial \mathbf{n}}\left(M_{0}\right) \geqslant \frac{\partial w}{\partial \mathbf{n}}\left(M_{0}\right)
$$

әде $\mathbf{n}=\mathbf{n}^{+}[\partial \Omega]-$ внутренняя нормаль.

Функцию $w$ из следствия 1 назовем приграничным нижним баръером для $v$. Недавно нам удалось выделить смысловое и конструктивное ядро этого барьера.

Определение 11. Назовем функцию $W m$-гессиановским ядром приграничного нижнего барьера в точке $M_{0} \in \partial \Omega$, если найдутся такие область $\Omega_{r}$ вида (39) и число $\nu>0$, что $W \in \mathbb{K}_{m}\left(\bar{\Omega}_{r}\right)$, причем

$$
W\left(M_{0}\right)=0,\left.\quad W\right|_{\partial \Omega_{r}} \leqslant 0,\left.\quad W\right|_{\partial \Omega_{r} \cap \Omega} \leqslant-\nu .
$$




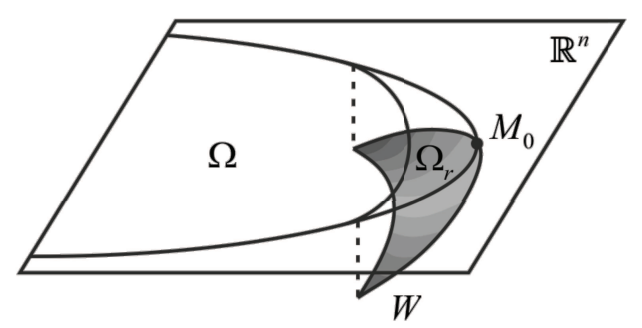

Рис. 1

Замечание 4. Поскольку $m$-допустимая функция $W$ не может иметь максимум в области $\Omega_{r}$, то условие $\left.W\right|_{\partial \Omega_{r}} \leqslant 0$ равносильно тому, что $W(x) \leqslant 0, x \in \bar{\Omega}_{r}$ (см. рис. 1$)$.

Легко убедиться, что если имеется $m$-гессиановское ядро $W$, то при достаточно большом $A>0$ условия следствия 1 будут выполнены для

$$
w=A W+\varphi,
$$

где $\varphi \in C^{2}\left(\Omega_{r}\right), v=\varphi$ на $\partial \Omega$.

Таким образом, оценка снизу производной по нормали для функции $v$, удовлетворяющей условию (37), сводится к проблеме существования $m$-гессиановских ядер приграничных нижних барьеров. Именно эту проблему можно считать генератором новых геометрических структур, которым посвящена данная работа. Почти весь дифференциально-геометрический аппарат, введенный в предыдущих разделах, концентрированно применяется при доказательстве следующей теоремы.

Теорема 3. Пусть $\partial \Omega-C^{2}$-гладкая гиперповерхность. Для того, чтобы в точке $M_{0} \in \partial \Omega$ существовало хотя бы одно и достаточно, чтобы поверхность $\partial \Omega$ была $(m-1)$-выпукла в этой точке.

Доказательство. Для начала создадим ситуацию, которая упрощает вычисление матрицы кривизны гиперповерхности $\partial \Omega \subset \mathbb{R}^{n}$ (см. раздел 3$)$.

Свяжем с точкой $M_{0}$ декартов базис $\left\{e_{1}, e_{2}, \ldots, e_{n}\right\}$, где $e_{n}=\mathbf{n}^{+}[\partial \Omega]\left(M_{0}\right)$ - внутренняя нормаль к $\partial \Omega$ в точке $M_{0}$. Тогда для поверхности $\partial \Omega$ имеется локальная параметризация

$$
X[\partial \Omega]=(\tilde{x}, \omega(\tilde{x}))^{T}, \quad \tilde{x}=\left(x^{1}, x^{2}, \ldots, x^{n-1}\right)^{T},
$$

с некоторой $C^{2}$-гладкой функцией $\omega(\tilde{x})$, причем $X\left(M_{0}\right)=\mathbf{0}$. Согласно лемме 1 в окрестности точки $M_{0}$ справедливы следующие формулы:

$$
\tau[\partial \Omega]=I-\frac{\omega_{\tilde{x}} \times \omega_{\tilde{x}}}{\sqrt{1+\omega_{\tilde{x}}^{2}}\left(1+\sqrt{1+\omega_{\tilde{x}}^{2}}\right)}, \quad \mathcal{K}[\partial \Omega]=\frac{\tau^{T} \omega_{\tilde{x} \tilde{x}} \tau}{\sqrt{1+\omega_{\tilde{x}}^{2}}} .
$$

Поскольку $\omega_{\tilde{x}}(\mathbf{0})=\mathbf{0}$, то

$$
\mathcal{K}[\partial \Omega]\left(M_{0}\right)=\omega_{\tilde{x} \tilde{x}}(\mathbf{0}) .
$$

I. Необходимость. Предположим, что $m$-гессиановское ядро $W$ существует. По определению 11 функция $-\left.W\right|_{\partial \Omega}=-W(\tilde{x}, \omega(\tilde{x}))$ имеет локальный минимум в точке $M_{0}$. Следовательно, ее матрица Гессе неотрицательно определена в точке $M_{0}$ :

$$
-W_{\tilde{x} \tilde{x}}(\mathbf{0})-W_{n}(\mathbf{0}) \mathcal{K}[\partial \Omega]\left(M_{0}\right) \in \bar{K}_{n} .
$$

Поскольку $W_{x x} \in K_{m}$, то по критерию Сильвестра (лемма 3 ) получаем, что $W_{\tilde{x} \tilde{x}} \in K_{m-1}$. Воспользуемся в точке $M_{0}$ монотонностью (28) функции $T_{m}$ :

$$
-W_{n}(\mathbf{0}) \mathcal{K}[\partial \Omega]\left(M_{0}\right) \in K_{m-1} .
$$


Из замечания 4 следует, что $W_{n}(\mathbf{0}) \leqslant 0$. Отсюда заключаем, что $\mathcal{K}[\partial \Omega]\left(M_{0}\right) \in K_{m-1}$ (и, кстати, $\left.W_{n}(\mathbf{0})<0\right)$.

II. Достаточность. Для доказательства достаточности мы предъявим $m$-гессиановское ядро $W$ в явном виде. Сначала сконструируем область (39). По условию теоремы матрица кривизны $\mathcal{K}[\partial \Omega]\left(M_{0}\right)$ является $(m-1)$-положительной, следовательно, имеется такое число $\varepsilon>0$, что

$$
\mathbf{k}_{m-1}[\partial \Omega]\left(M_{0}\right) \geqslant 3 \varepsilon .
$$

В силу непрерывности найдется достаточно малое число $r>0$, для которого гиперповерхность

$$
\Gamma_{r}^{0}=\left\{|\tilde{x}| \leqslant r, x^{n}=\omega(\tilde{x})\right\} \subset \partial \Omega
$$

является $(m-1)$-выпуклой и $\mathbf{k}_{m-1}\left[\Gamma_{r}^{0}\right] \geqslant 2 \varepsilon$.

Введем гиперповерхность

$$
\Gamma_{r}^{\beta}=\left\{|\tilde{x}| \leqslant r, x^{n}=\hat{\omega}(\tilde{x})\right\}, \quad \hat{\omega}(\tilde{x})=\omega(\tilde{x})+\frac{\beta}{2}\left(r^{2}-\tilde{x}^{2}\right), \quad \beta>0 .
$$

Поверхность $\Gamma_{r}^{\beta}$ располагается внутри области $\Omega$ (рис. 2), кроме края, который совпадает с краем $\Gamma_{r}^{0}$.

Заметим, что поверхность $\Gamma_{r}^{0}$, будучи частью замкнутой поверхности $\partial \Omega$, ориентирована внутренней нормалью. Ориентируем так же и поверхность $\Gamma_{r}^{\beta}$, т.е. выбираем для нее направление нормали, образующее острый угол с ортом $e_{n}$.

Поскольку $\Gamma_{r}^{\beta}$ отличается от $\Gamma_{r}^{0}$ на незначительное квадратичное слагаемое, то существует такое число $\beta_{0}>0$, что для всех $0<\beta \leqslant \beta_{0}$ поверхность $\Gamma_{r}^{\beta}$ оказывается также $(m-1)$-выпуклой и

$$
\mathbf{k}_{m-1}\left[\Gamma_{r}^{\beta}\right] \geqslant \varepsilon .
$$

Действительно, матрица $\mathcal{K}\left[\Gamma_{r}^{\beta}\right]$, как и $\mathcal{K}\left[\Gamma_{r}^{0}\right]$, вычисляется по формулам $(41),(42)$ с подстановкой $\hat{\omega}$ вместо $\omega$. Прямой расчет приводит к выводу, что

$$
\mathbf{k}_{m-1}\left[\Gamma_{r}^{\beta}\right]=\mathbf{k}_{m-1}\left[\Gamma_{r}^{0}\right]+O(\beta) .
$$

В качестве приграничной области (39) рассмотрим область, ограниченную поверхностями $\Gamma_{r}^{0}$ и $\Gamma_{r}^{\beta}$, и запишем ее при помощи вспомогательной функции $y(x)$ :

$$
\Omega_{r}^{\beta}=\left\{x \in \Omega: \frac{\beta}{2} \tilde{x}^{2}<y(x)<\frac{\beta}{2} r^{2}, y=x^{n}-\omega(\tilde{x})+\frac{\beta}{2} \tilde{x}^{2}\right\} .
$$

Параметр $\beta \leqslant \beta_{0}$ будет еще раз уточнен далее.

Отметим, что несмотря на $(m-1)$-выпуклость $\Gamma_{r}^{0}$ и $\Gamma_{r}^{\beta}$, замкнутая поверхность $\partial \Omega_{r}^{\beta}=\Gamma_{r}^{0} \cup \Gamma_{r}^{\beta}$ не является $(m-1)$-выпуклой, так как $\Gamma_{r}^{\beta}$ ориентирована не внутренней по отношению к $\Omega_{r}^{\beta}$, а внешней нормалью.

Рассмотрим в области (43) функцию

$$
W=\frac{y}{\beta r^{2}}\left(\frac{y}{2 \beta r^{2}}-1\right) .
$$

Легко видеть, что для этой функции выполнены все условия (40) с $\nu=3 / 8$.

Осталось проверить, что $W \in \mathbb{K}_{m}\left(\bar{\Omega}_{r}^{\beta}\right)$. Фиксируем произвольно выбранную точку $x \in \Omega_{r}^{\beta}$ и вычисляем:

$$
W_{x}=\frac{y-\beta r^{2}}{\beta^{2} r^{4}} y_{x}, \quad W_{x x}=\frac{1}{\beta^{2} r^{4}}\left(\left(\beta r^{2}-y\right)\left(-y_{x x}\right)+y_{x} \times y_{x}\right) .
$$

Учитывая неравенство $\beta r^{2}-y \geqslant 0$ в $\Omega_{r}^{\beta}, m$-однородность функций $T_{m}$ и соотношение $(29)$, вычислим значение оператора $T_{m}[W]=T_{m}\left(W_{x x}\right)$ :

$$
T_{m}[W]=\frac{\left(\beta r^{2}-y\right)^{m-1}}{\left(\beta^{2} r^{4}\right)^{m}}\left(\left(\beta r^{2}-y\right) T_{m}[-y]+T_{m}^{i j}\left(-y_{x x}\right) y_{i} y_{j}\right)
$$




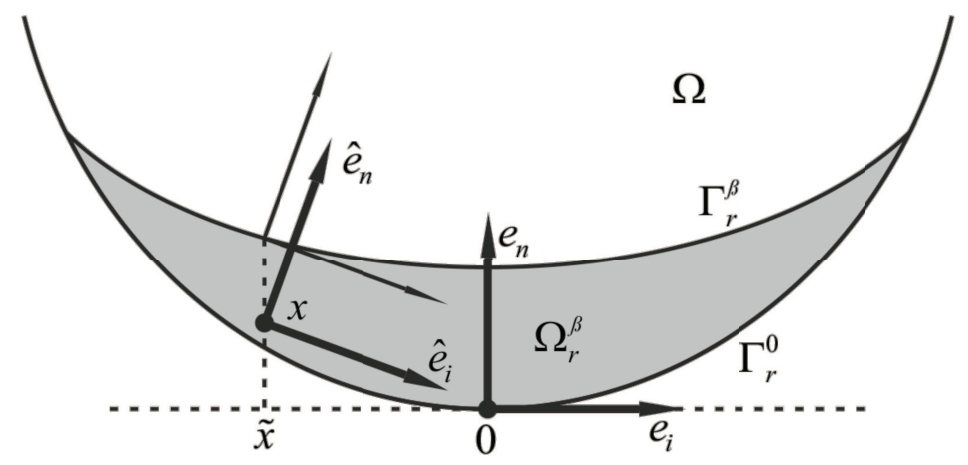

Рис. 2

Благодаря ортогональной инвариантности $m$-следа (свойство (25)), левая часть равенства (46) и первое слагаемое в правой части не зависят от выбора декартовой системы координат в пространстве $\mathbb{R}^{n}$. Следовательно, и второе слагаемое не зависит - именно оно играет ключевую роль. Для удобства его вычисления введем в данной точке $x=\left(\tilde{x}, x^{n}\right) \in \Omega_{r}^{\beta}$ новый декартов базис, порожденный сопровождающим базисом (11) к поверхности $\Gamma_{r}^{\beta}$ в точке $\tilde{x}$. Именно, положим

$$
\hat{e}_{i}(x)=X_{(i)}\left[\Gamma_{r}^{\beta}\right](\tilde{x}), \quad i=1,2, \ldots, n-1, \quad \hat{e}_{n}(x)=\mathbf{n}\left[\Gamma_{r}^{\beta}\right](\tilde{x})
$$

(см. рис. 2).

Поскольку $\Gamma_{r}^{\beta}$ является поверхностью уровня функции $y$, то в данной точке $x$ имеем

$$
\frac{\partial y}{\partial \hat{x}^{n}}(x)=\left|y_{x}\right|=\sqrt{1+\hat{\omega}_{x}^{2}}, \quad \frac{\partial y}{\partial \hat{x}^{k}}(x)=0, \quad k=1,2, \ldots, n-1 .
$$

Поэтому

$$
T_{m}^{i j}\left(-y_{x x}\right) y_{i} y_{j}=\left(1+\hat{\omega}_{x}^{2}\right) T_{m}^{n n}\left(-y_{\hat{x} \hat{x}}\right)
$$

Легко проверить, что

$$
T_{m}^{n n}\left(-y_{\hat{x} \hat{x}}\right)=T_{m-1}\left(-y_{\hat{x} \hat{x}}^{\langle n\rangle}\right),
$$

где символом $-y_{\hat{x} \hat{x}}^{\langle n\rangle} \in \operatorname{Sym}(n-1)$ обозначена матрица, полученная из матрицы $-y_{\hat{x} \hat{x}} \in \operatorname{Sym}(n)$ вычеркиванием строки и столбца с номером $n$. Вычисляем в данной точке $x$ :

$$
-\frac{\partial^{2} y}{\partial \hat{x}^{k} \partial \hat{x}^{l}}=-\left(y_{x x} \hat{e}_{k}, \hat{e}_{k}\right)=\tau^{T} \hat{\omega}_{\tilde{x} \tilde{x}} \tau, \quad k, l=1,2, \ldots, n-1, \quad \tau=\tau\left[\Gamma_{r}^{\beta}\right](\tilde{x}) .
$$

По формуле (42), переписанной для поверхности $\Gamma_{r}^{\beta}$, получаем

$$
-y_{\hat{x} \hat{x}}^{\langle n\rangle}=\sqrt{1+\hat{\omega}_{x}^{2}} \mathcal{K}\left[\Gamma_{r}^{\beta}\right](\tilde{x}) .
$$

По ранее гарантированным свойствам поверхности $\Gamma_{r}^{\beta}$ имеем окончательную оценку в точке $x$ :

$$
T_{m}^{i j}\left(-y_{x x}\right) y_{i} y_{j}=\left(1+\hat{\omega}_{x}^{2}\right)^{(m+1) / 2} \mathbf{k}_{m-1}\left[\Gamma_{r}^{\beta}\right](\tilde{x}) \geqslant \varepsilon .
$$

Теперь рассмотрим соотношение (46), учитывая (47) и $\left|\beta r^{2}-y\right| \leqslant \beta r^{2}$ в $\bar{\Omega}_{r}^{\beta}$. Приходим к выводу, что существует такое число $\beta_{1}>0$, что $T_{m}[W]>0$ в области $\bar{\Omega}_{r}^{\beta}$ для всех $\beta \leqslant \beta_{1} \leqslant \beta_{0}$.

Наконец, рассмотрим матрицу $W_{x x}$ в точке $M_{0} \in \partial \Omega$. Напомним, что в исходной системе координат ей соответствует точка $\mathbf{0}$. Вычисляем

$$
W_{\tilde{x} \tilde{x}}(\mathbf{0})=\frac{\beta r^{2}-y}{\beta^{2} r^{4}} \hat{\omega}_{\tilde{x} \tilde{x}}(\mathbf{0})=\frac{\beta r^{2}-y}{\beta^{2} r^{4}} \mathcal{K}\left[\Gamma_{r}^{\beta}\right](\mathbf{0}) .
$$


Следовательно, $W_{\tilde{x} \tilde{x}}(\mathbf{0}) \in K_{m-1}$, что вместе с $T_{m}\left(W_{x x}\right)>0$ означает $W_{x x}(\mathbf{0}) \in K_{m}$ (критерий Сильвестра, лемма 3). Поскольку $T_{m}[W]>0$ в области $\bar{\Omega}_{r}^{\beta}$, то с учетом леммы 5 приходим к выводу, что $W \in \mathbb{K}_{m}\left(\bar{\Omega}_{r}^{\beta}\right)$. Таким образом, искомое ядро барьера построено.

В заключение отметим, что наше внимание было сконцентрировано на построении $m$-гессиановских нижних барьеров по той причине, что верхний барьер достается гораздо проще. Например, для функции $u \in \mathbb{K}_{m}(\Omega)$ верхним барьером является любая гармоническая функция $w$, совпадающая с $u$ на границе области $\Omega$. Действительно, в этом случае $(u-w) \in \mathbb{K}_{1}(\Omega)$, а значит, функция $(u-w)$ не может достигать максимума внутри $\Omega$.

\section{СПИСОК ЛИТЕРАТУРЫ}

1. Зорич В. А. Математический анализ. - М.: МЦНМО, 2015.

2. Ивочкина H. М. Интегральный метод барьерных функций и задача Дирихле для уравнений с операторами типа Монжа-Ампера// Мат. сб. - 1980. - 112 (154), № 2 (6). - С. 193-206.

3. Ивочкина H. М. Описание конусов устойчивости, порождаемых дифференциальными операторами типа Монжа-Ампера// Мат. сб. - 1983. - 122 (164), № 2 (10). - С. 265-275.

4. Ивочкина H. М. Задача Дирихле для уравнения кривизны порядка $m / /$ Алгебра и анализ. - 1990. 2, № 3. - C. 192-217.

5. Ивочкина Н. М. От конусов Гординга к $p$-выпуклым гиперповерхностям// Совр. мат. Фундам. напр. - 2012. - 45. - C. 94-104.

6. Ивочкина Н. М., Ладыженская О. А. Оценка вторых производных на границе для поверхностей, эволюционирующих под действием главных кривизн// Алгебра и анализ. - 1997. - 9, № 2. - С. 3050.

7. Ивочкина Н. М., Филимоненкова Н. В. О новых структурах в теории полностью нелинейных уравнений// Совр. мат. Фундам. напр. - 2015. - 58. - С. 82-95.

8. Ивочкина Н. М., Филимоненкова Н. В. Геометрические модели в теории нелинейных дифференциальных уравнений. - СПб. мат. об-во, 2016.

9. Крылов $H$. В. Ограниченно неоднородные эллиптические и параболические уравнения в области// Изв. АН СССР. Сер. мат. - 1983. - 47, № 1. - С. 75-108.

10. Погорелов A. В. Многомерная проблема Минковского. - М.: Наука, 1975.

11. Сафонов М. В. Неравенство Харнака для эллиптических уравнений и гельдеровость их решений// Зап. науч. семин. ЛОМИ. - 1983. - № 12. - С. 272-287.

12. Филимоненкова H. В. О классической разрешимости задачи Дирихле для невырожденных $m$ гессиановских уравнений// Пробл. мат. анал. - 2011. - № 60. - С. 89-111.

13. Филимоненкова Н. В. Критерий Сильвестра для $m$-положительных матриц. - СПб. мат. об-во, 2014.

14. Филимоненкова Н. В., Бакусов П. А. Гиперболические многочлены и конусы Гординга// Мат. просв. Cep. 3. - 2016. - № 20. - C. 143-166.

15. Филимоненкова Н. В., Бакусов П. А. Анализ $m$-выпуклости многомерных параболоидов и гиперболоидов// Мат. просв. Сер. 3. - 2017. - № 21. - С. 64-86.

16. Caffarelly L., Nirenberg L., Spruck J. The Dirichlet problem for nonlinear second order elliptic equations, III. Functions of the eigenvalues of the Hessian// Acta Math. - 1985. — 155. - P. 261-301.

17. Caffarelli L., Nirenberg L., Spruck J. Nonlinear second-order elliptic equations, V. The Dirichlet problem for Weingarten hypersurfaces// Commun. Pure Appl. Math. — 1988. — 41. — P. 47-70.

18. Evans L. C. Classical solutions of fully nonlinear convex second order elliptic equations// Commun. Pure Appl. Math. - 1982. - 25. - P. 333-363.

19. Gårding L. An inequality for hyperbolic polynomials// J. Math. Mech. - 1959. — 8. - P. 957-965.

20. Ivochkina N. M. Geometric evolution equations preserving convexity// Adv. Math. Sci. - 2007. - 220. P. 191-121.

21. Ivochkina N. M. Symmetry and geometric evolution equations// Записки науч. сем. ПОМИ. - 2004. 318. - C. $135-146$.

22. Ivochkina N. M., Filimonenkova $N$. V. On the bakgrounds of the theory of $m$-Hessian equations// Commun. Pure Appl. Anal. — 2013. - 12, № 4. - P. 1687-1703.

23. Ivochkina N. M., Filimonenkova N. V. On algebraic and geometric conditions in the theory of Hessian equations// J. Fixed Point Theory Appl. — 2015. - 16, № 1. - P. 11-25. 
24. Ivochkina N. M., Filimonenkova N. V. Attractors of $m$-Hessian evolutions// J. Math. Sci. -2015 . -207 , № 2. - P. 226-235.

25. Ivochkina N. M., Nehring Th., Tomi F. Evolution of starshaped hypersurfaces by nonhomogeneous curvature functions// Алгебра и анализ. - 2000. - 12, № 1. - С. 185-203.

26. Ivochkina N. M., Yakunina G. V., Prokof'eva S. I. The Gårding cones in the modern theory of fully nonlinear second order differential equations// J. Math. Sci. — 2012. - 184, № 3. - P. 295-315.

27. Trudinger N. S. The Dirichlet problem for the prescribed curvature equations// Arch. Rat. Mech. Anal. 1990. - 111. - P. 153-179.

28. Trudinger N. S. On the Dirichlet problem for Hessian equations// Acta Math. - 1995. - 175. - P. 151164.

29. Wang X. J. The $k$-Hessian equation// Lect. Notes Math. — 2009. — 1977. — P. 177-252.

30. Urbas $J$. On the expansion of starshaped hypersurfaces by symmetric functions of their principal curvatures// Math. Z. - 1990. - 205. - P. 355-372.

Филимоненкова Надежда Викторовна

Санкт-Петербургский политехнический университет Петра Великого

E-mail: nf33@yandex.ru 